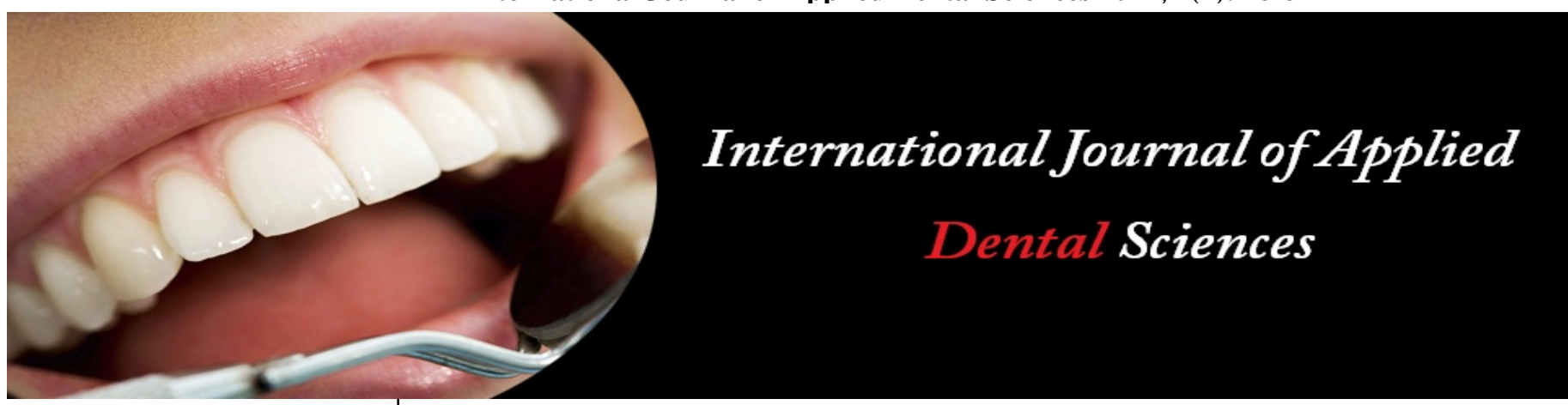

ISSN Print: 2394-7489

ISSN Online: 2394-7497

IJADS 2021; 7(4): 78-81

(C) 2021 IJADS

www.oraljournal.com

Received: 07-08-2021

Accepted: 09-09-2021

Dr. Ravi Sagar Singh Shahi Department of Prosthodontics, Faculty of Dental Sciences, SGT University, Gurugram, Haryana, India

Dr. Chahat Saini

Department of Prosthodontics, Faculty of Dental Sciences, SGT University, Gurugram, Haryana, India

Corresponding Author:

Dr. Ravi Sagar Singh Shahi

Department of Prosthodontics,

Faculty of Dental Sciences, SGT

University, Gurugram, Haryana,

India

\section{A new clinical approach in fabrication of closed hollow bulb obturator: A case report}

\section{Dr. Ravi Sagar Singh Shahi and Dr. Chahat Saini}

DOI: https://doi.org/10.22271/oral.2021.v7.i4b.1354

\section{Abstract}

Obturators are the most common treatment modality compared to surgical reconstruction for Maxillectomy in oral cancer patients. The obturators frequently become bulky and need to be hollowed out properly in the defect area to decrease weight and make them comfortable for the patient. This case report describes the technique of using a hollow bulb obturator on an adult patient who had undergone a maxillectomy. An obturator was fabricated with the use of a self-cured acrylic resin shim filled with wax in the defect area during the packing procedure of the prosthesis, and the wax was eliminated after curing. It is a single step process for the closed-hollow bulb obturator fabrication as a single unit, with uniform wall thickness around the hollow space, ensuring the least possible weight of the hollow obturator. This technique attains the probable internal facet of the hollow space with a uniform wall thickness of the prosthesis.

Keywords: closed hollow bulb, curing, maxillectomy, obturator, oral cancer, self cure acrylic resin, wax

\section{Introduction}

In postmaxillectomy patients, the Obturator is critical for functional rehabilitation and appropriate sealing defect ${ }^{[1]}$. The unwanted weight due to bulk in the defect portion becomes a challenge as it hampers the retention, stability and support of the prosthesis. It ultimately leads to traumatic functional occlusion and improper oroantral or oronasal seal ${ }^{[2]}$. Various processing techniques have been described in the literature to fabricate a water-tight closedhollow obturator with a durable material ${ }^{[3]}$.

Previously used techniques are much more technique sensitive, and so a simplified technique was needed for the fabrication of the prosthesis. This paper presents a simplified technique for the fabrication of a closed hollow bulb obturator as a single unit with uniform wall thickness around the hollow space, ensuring the least possible weight using self-curing acrylic resin and wax.

\section{Case Report}

A 70-year-old male patient was referred to the Department of Prosthodontics for prosthetic rehabilitation after surgical removal of Mucoepidermoid carcinoma from the right side of maxilla with a chief complaint of inability to chew food, nasal spewing of fluids, compromised esthetics, difficulty in speech with exaggerated nasality in his voice. Facial disproportion with a depressed right malar region was seen extraorally (Figure1). A healthy postmaxillectomy defect involving part of the posterior hard palate, alveolar ridge, maxillary tuberosity, and some parts of the soft palate on the right side with compromised 23,24,25,26 with a partially edentulous mandibular arch was seen intraorally in the patient (Figure 2). A hollow bulb obturator for the maxillary arch and RPD for the mandibular arch were planned for the prosthetic rehabilitation of this patient. A fixed treatment plan was delayed for a period of one year to avoid the chances of osteoradionecrosis as the patient had undergone radiotherapy. 


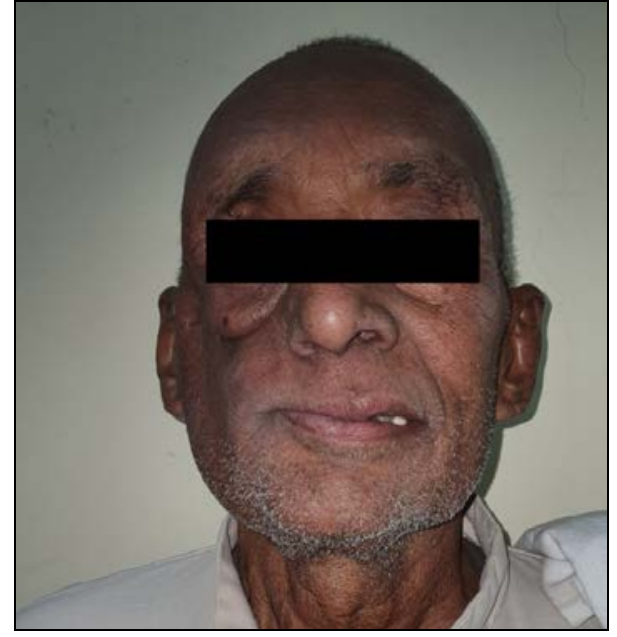

Fig 1: Extraoral view

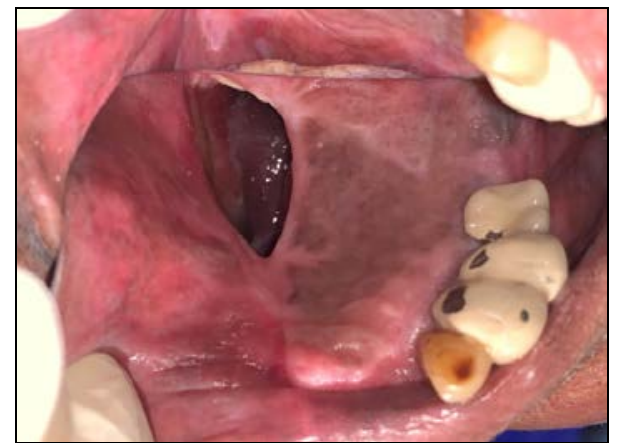

Fig 2: Intaoral view showing the maxillary defect

\section{Procedure:}

Prior to impression making, proper tray selection was done, and the connection between the defect and the nasal cavity was blocked using a gauze piece tied with a thread to avoid the entrance of impression material into it. A primary impression was made using Alginate (Zelgan 2002 dust-free easy mixing, DENTSPLY India Pvt. Ltd., Haryana) (Figure 3). The custom tray fabrication using autopolymerizing acrylic resin (self-curing acrylic repair material, DENTSPLY India Pvt. Ltd., India) (Figures 4) and border moulding using green stick impression compound (DPI Pinnacle, tracing stick, Dental Products of India, Mumbai) was done, and the final impression was made using light viscosity addition silicone impression material and pickup impression was made using Alginate (Figures 5). The master cast was poured using die stone (Ultrarock, Kalabhai Karson Pvt. Ltd., India).

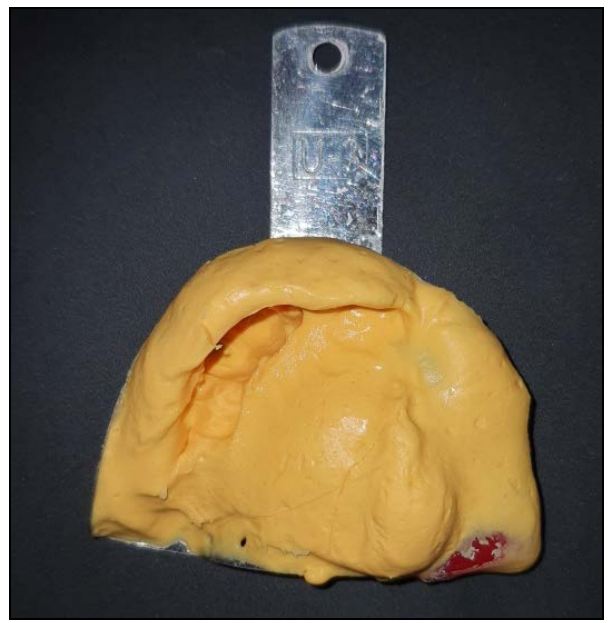

Fig 3: Primary impression

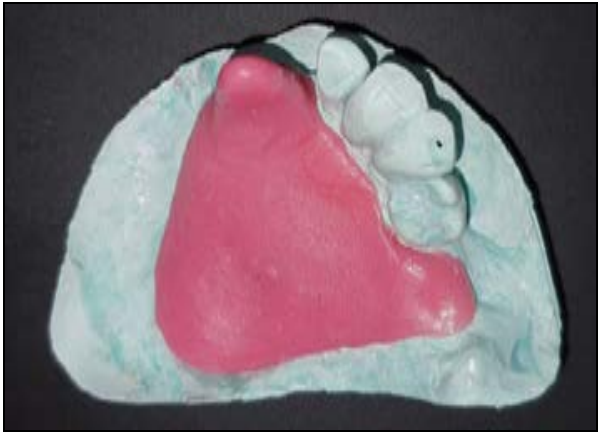

Fig 4: Self cure acrylic resin custom tray

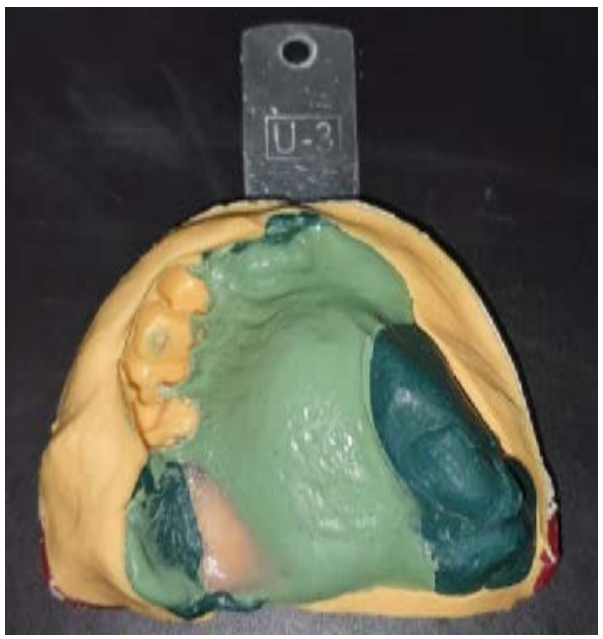

Fig 5: Final Impression

After the trial insertion appointment, the wire clasps were made on the cast for additional retentive purposes of the obturator, and the surrounding acrylic record base was trimmed from that area where the clasps were reseated. The master cast along with the trial prosthesis was invested in the flask after wax up and the flask was kept under a mechanical clamp. Dewaxing was done in a conventional manner and was left to cool down.

Before the packing procedure, separating medium was applied in the defect area and a thin self-curing acrylic resin was loaded into the area and allowed to set (Figure 6). After setting, it was removed from the defect and thinned from the inner surface (Figure 7). It was again seated in its position in the defect area and then Vaseline was applied to its inner surface and was filled with wax up to the upper surface of the defect area, then again Vaseline was applied to the wax surface (Figure 8).

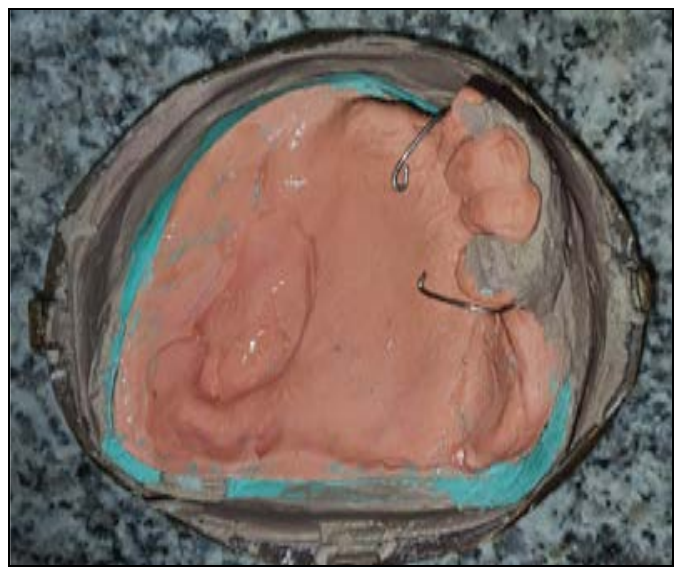

Fig 6: Separating medium applied to the defect area 


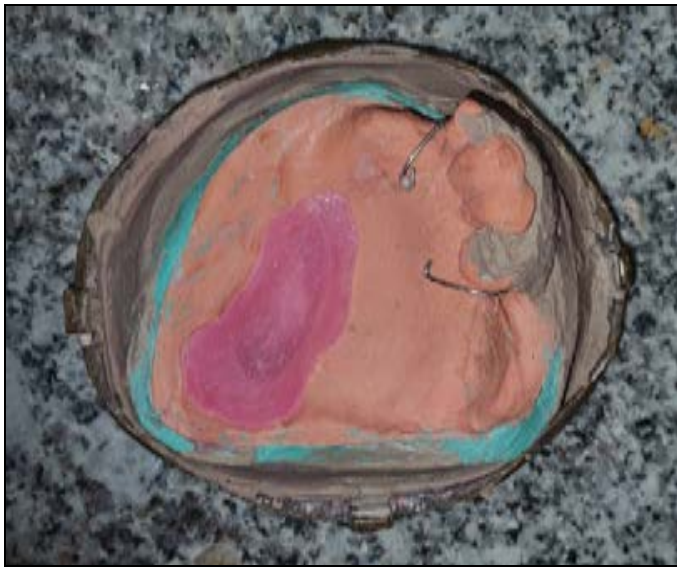

Fig 7: A self-curing acrylic shim that has been thinned out and reseated in the defect area.

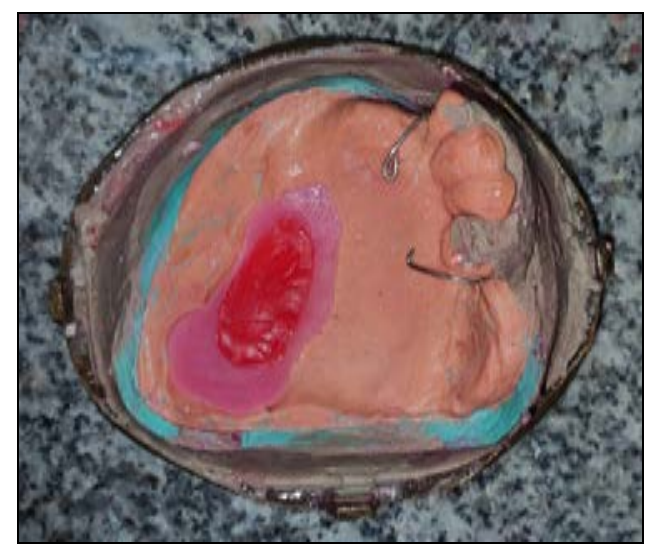

Fig 8: Wax filled in the self-curing acrylic resin shim in the defect area

The packing was done using heat cure acrylic resin (Trevalon Denture Material, DENTSPLY India Pvt. Ltd., India). The resin material was mixed and packed into the flask and closed under the mechanical clamp. The assembly was kept at room temperature for 2 hours and the curing cycles were performed as per the manufacturer instructions. The clamp-flask assembly was kept at room temperature for 24 hours after curing (bench cooling). The flasks were opened after bench cooling. Two holes in the intaglio surface of the prosthesis in the defect area were made using a round carbide bur. Wax was removed by using a hot water bath, and the forceful stream of hot water was applied through one of the holes to remove the remaining wax through the other hole, forming a hollow space in the shape of the wax-bolus (Figure 9).

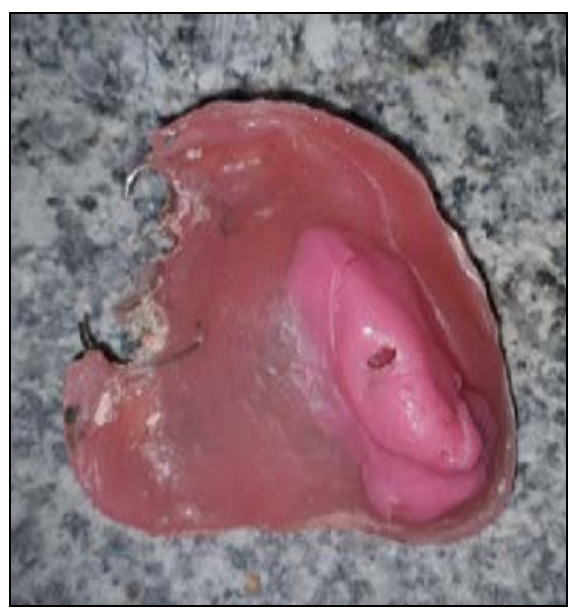

Fig 9: Wax elimination by making holes

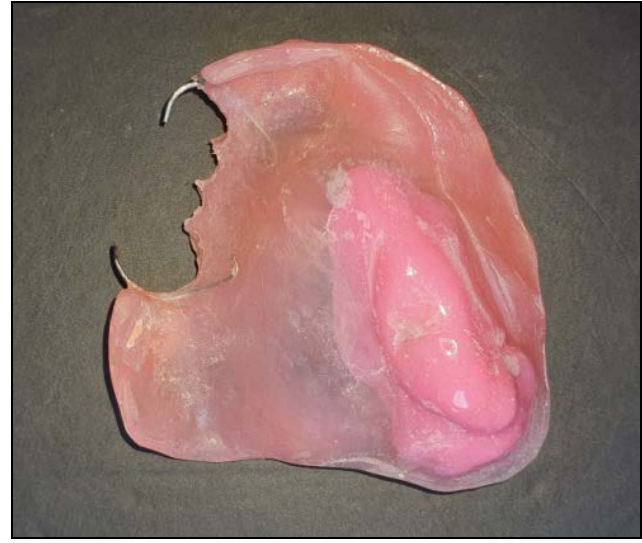

Fig 10: Holes sealed

The holes were sealed using Autopolymerizing acrylic resin (Figure 10). Finishing and polishing procedures of the prosthesis were done (Figure 11). The obturator was inserted in the patient's mouth (Figure 12). The patient was happy and content with his enhanced function, speech, and aesthetics (Figure 13). Postinsertion instructions with regard to prosthesis care and insertion were given to the patient. The patient was also instructed to follow up appointments at an interval of 3 months for a period of 1 year. The patient reported no complaints during the follow-up appointments.
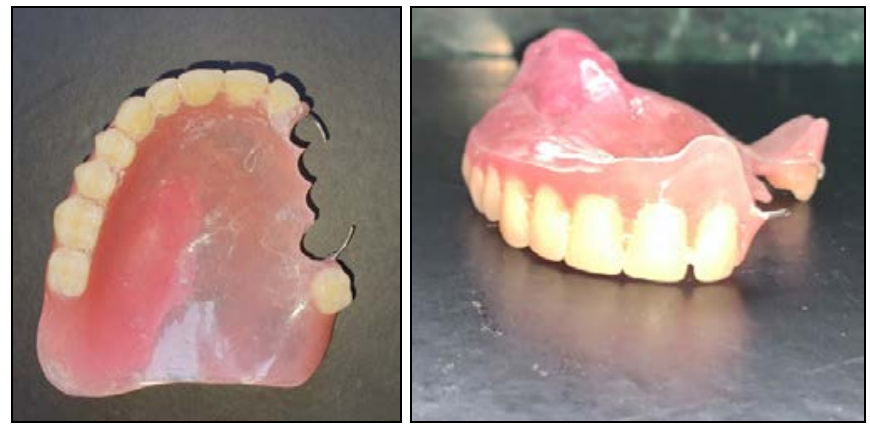

Fig 11: Final Prosthesis, Completed and Polished

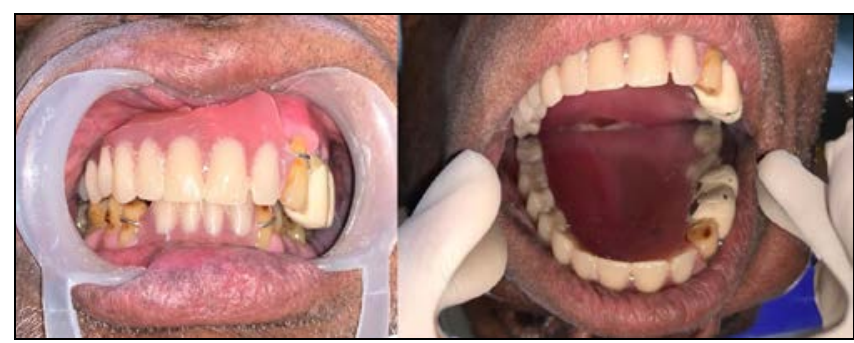

Fig 12: Intraoral View

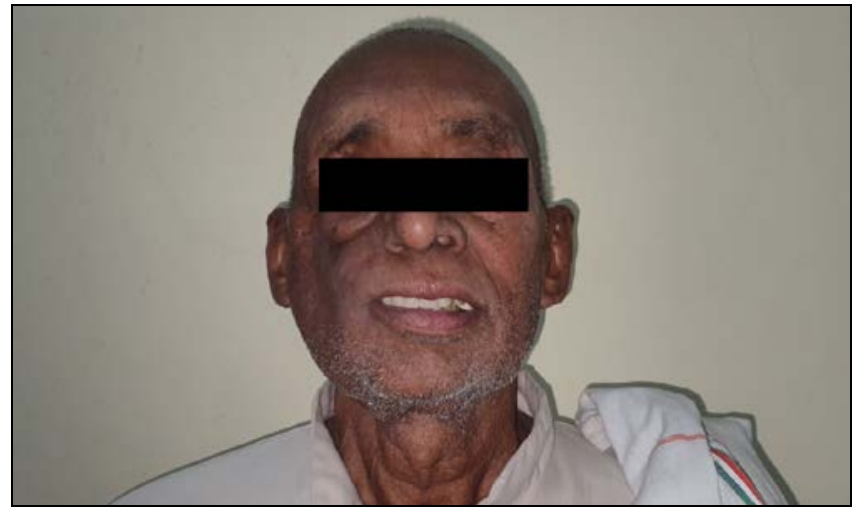

Fig 13: Extraoral view 


\section{Discussion}

Patients with maxillectomy defects present a noteworthy challenge for prosthetic rehabilitation, mainly in retention of the prosthesis due to its bulk, which in turn poses difficulty in speech and mastication. Several techniques and materials have been presented earlier to fabricate a lightweight, hollow obturator. trimming out the unwanted part directly after processing ${ }^{[4]}$, closure of the superior border of the defect in the prosthesis with a lid ${ }^{[5]}$, incorporating materials like sugar $^{6}$ and ice ${ }^{[7]}$ during packing. Separate fabrication of two halves and joining them with an autopolymerizing resin or two step processing technique ${ }^{[8,9]}$, using preformed plastic shapes ${ }^{[10]}$, plaster matrix ${ }^{[11]}$, resin shim ${ }^{[12]}$ and polyurethane foam ${ }^{[13]}$ are also described in the literature.

The technique described in this article is superior to all other techniques previously described in two ways: (1) The entire prosthesis is a single unit, and (2) the size and shape of the hollow space achieved allow for uniform wall thickness in the closed hollow obturator. This technique is a variation of some previously described techniques which comprise the use of a preshaped wax-bolus to maintain a predictable internal dimension of a hollow space ${ }^{[14]}$. Some of the issues regarding the technique should be carefully handled to achieve the best possible results. (1) Prior to final curing of the prosthesis, the self-curing acrylic resin shim should be properly seated in the defect area. (2) To elude wax distortion in the curing process, bench cure after packing is necessary. (3) Make sure the waxbolus is completely eliminated from the bulb of the prosthesis.

\section{Conclusion}

A number of qualities can be credited to the usage of this procedure for fabrication of closed hollow bulb obturators. The prosthesis is fabricated by means of a single flask, which significantly decreases the laboratory time and makes the technique simple. The thickness of the hollow bulb can be controlled and light-weight closed-bulb prosthesis can be fabricated, avoiding the chance of water leakage and bacterial infection.

\section{References}

1. Ono T, Kohda H, Hori K, Nokubi T. "Masticatory performance in postmaxillectomy patients with edentulous maxillae fitted with obturator prostheses," The International Journal of Prosthodontics 2007;20(2):145-150.

2. Taylor TD. Clinical Maxillofacial Prosthetics, Quintessence Publishing 2000.

3. Patil PG, Patil SP. A hollow definitive obturator fabrication technique for management of partial maxillectomy. J Adv Prosthodont 2012;4:248-53.

4. Habib BH, Driscoll CF. Fabrication of a closed hollow obturator. J Prosthet Dent 2004;91(4):383-385.

5. Sridevi JR, Kalavathy N, Jayanthi N, Manjula N. Techniques for fabricating hollow obturator: Two case reports. SRM J Res Dent Sci 2014;5:143-6.

6. Parel SM, LaFuente H. Single-visit hollow obturators for edentulous patients. J Prosthet Dent 1978;40:426-9.

7. Schneider A. Method of fabricating a hollow obturator. J Prosthet Dent 1978;40:351.

8. Browning JD, Kinderknecht J. Fabrication of a hollow obturator with fluid resin. J Prosthet Dent 1984;52(6):891-895.

9. Elangovan S, Loibi E. Two-piece hollow bulb obturator. Indian J Dent Res 2011;22(3):486-488.

10. el Mahdy AS. Processing a hollow obturator. J Prosthet
Dent 1969;22:682-6.

11. Asher ES, Psillakis JJ, Piro JD, Wright RF. Technique for quick conversion of an obturator into a hollow bulb. J Prosthet Dent 2001;85:419-20.

12. Chalian VA, Barnett MO. A new technique for constructing a one-piece hollow obturator after partial maxillectomy. J Prosthet Dent 1972;28:448-53.

13. Tanaka Y, Gold HO, Pruzansky S. A simplified technique for fabricating a lightweight obturator. J Prosthet Dent 1977;38:638-42.

14. Patil PG, Patil SN. Lost wax-bolus technique to process closed hollow obturator with uniform wall thickness using single flasking procedure. J Indian Prosthodont Soc 2017; $17: 84-8$ 